\title{
INTERFERÊNCIA DO INTERVALO DE ADMINISTRAÇÃO DA DROGA SOBRE A NEFROTOXICIDADE DA GENTAMICINA EM RATOS.
}

\author{
INFLUENCE OF THE DOSE REGIMEN ON THE GENTAMICIN \\ NEPHROTOXICITY IN RATS.
}

\author{
Verônica Cunha Rodrigues Oliveira** \\ Carla Roberta Tejos* \\ Elisabeth Mie Hosaka* \\ Sara Cogo Andrade* \\ Magali Araújo*** \\ Maria de Fátima Fernandes Vattimo****
}

OLIVEIRA, VCR et al. Interferência do intervalo de administração da droga sobre a nefrotoxicidade da gentamicina em ratos, Rev Esc Enf USP, v. 35, n.2, p. 200-4, jun, 2001

\section{RESUMO}

A insuficiencia renal aguda (IRA) que apresenta indice de mortalidade em torno de 50\%, pode ser definida como um abrupto declinio da filtração glomerular, resultante de isquemia ou toxicidade. A nefrotoxicidade por drogas é uma das etiologias mais freqüentes (27\%) e sugere-se que o intervalo de administração da droga pode interferir neste efeito colateral, entretanto o melhor regime de administração ainda não está bem estabelecido. Este conhecimento proporcionaria uma atuação mais direcionada de enfermagem na prevenção desta IRA hospitalar. Os resultados obtidos nesta pesquisa, indicam que a infusão única de gentamicina determina menor nefrotoxicidade, provavelmente devido à redução da sua concentração plasmática nas $24 \mathrm{hs}$, diminuindo o acúmulo intracelular deste fármaco, um dos principais mecanismos celulares deste tipo de lesão. Este regime de tratamento mostra portanto vantagens quanto ao custo, efeito nefrotóxico e segurança quanto à eficácia terapêutica.

PALAVRAS-CHAVE: Insuficiência renal aguda. Nefrologia. Antibióticos.

\begin{abstract}
The acute renal failure (ARF), that still presents a righ mortality rate (50\%) can be defined as an abrupt decline of the glomerular filtration, resultant of isquemic or toxicity event. The drugs nephrotoxicity is one of the most frequent cause (27\%) of ARF and it is suggested that the interval of administration of the drug can interfere in this side effect, however the best administration regimen is not very well established. This study evaluated the renal function of rats that received gentamicin $(100 \mathrm{mg} / \mathrm{kg})$ in one dose or in two doses $(2 x 50 \mathrm{mg} / \mathrm{kg})$,by intraperitoneal infusion. The results obtained in this research, indicated that the single infusion of gentamicin determined smaller nephrotoxicity by the reduction of serum concentration of this drug in 24 hours, decreasing the intracelular accumulation of this gentamicin, which is one of the main cellular mechanisms of this renal injury. The single dose treatment regime, otherwise, showes advantages not only related to the nephrotoxicity effect, but also it is relevant to the cost and safety, which can be rationable factors in the administration of this drug.
\end{abstract}

KEYWORDS: Kidney failure acute. Nephrology. Antibiotics.

* $\quad$ Alunas da Graduação em Enfermagem da EEUSP, formadas em 2000. Iniciação Científica 1999-2000.

** Aluna do Programa de Pós-Graduação em Enfermagem em Saúde do Adulto da EEUSP, nível Mestrado.

*** Aluna do Programa e Pós-Graduação em Medicina, área de Nefrologia da FMUSP, nível doutorado

**** Professora Doutora do Departamento de Enfermagem Médico-Cirúrgica da EEUSP, coordenadora do Laboratório Experimental da EEUSP. 


\section{INTRODUÇÃO}

A insuficiência renal aguda (IRA) pode ser definida como um abrupto declínio da filtração glomerular resultante de isquemia ou toxicidade, que inclui a diminuição da permeabilidade capilar glomerular, obstrução tubular e vasoconstrição intra-renal, São comprometimentos celulares considerados sub-letais e letais ${ }^{20}$, devido ao acúmulo no organismo de compostos nitrogenados como uréia e creatinina, mas que podem ser reversiveis ao contrário da insuficiência renal crônica.

A IRA ocorre de forma abrangente na população, sendo desencadeada por diversas causas. Encontra-se associada à elevada mortalidade, em torno de $50 \% 5$, o que faz com que as pesquisas sejam indispensáveis à caminho de um efetivo controle,

A busca de medidas de prevenção contra o desenvolvimento da IRA é essencial, e provavelmente mais efetiva, para diminuir tal indice de mortalidade do que tratamentos utilizados após o seu desenvolvimento ${ }^{5}$,

Atualmente uma das etiologias mais freqüentes de IRA é a nefrotoxicidade por drogas (27\%), indice este compativel com uma crescente tendência mundial ao longo dos últimos trinta anos ${ }^{5}$,

Dentre as drogas nefrotóxicas precursoras da IRA temos os aminoglicosídeos com elevada incidência, atingindo aproximadamente $20 \%$ dos pacientes submetidos a essa terapêutica, podendo chegar a níveis alarmantes de $50 \%$ em terapias instituídas por mais de duas semanas 8,12,17,24 São antibióticos de largo expectro (gentamicina, tobramicina, neomicina, amicacina, netilmicina), extremamente eficazes nas infecções por gram-negativos, com atividades também contra microrganismos gram-positivos $21,24,25$

Dentre as diversas variáveis que concernem as discussões sobre a nefrotoxicidade dos aminogli-cosídeos, temos a configuração da molécula dos mesmos como uma considerável determinante 25 . Quando na constituição da molécula da droga há um maior número de grupamentos amina é atribuído ao fármaco maior toxicidade sobre o rim. Assim, verifica-se maior toxicidade renal da gentamicina quando comparada a outras drogas, como por exemplo a tobramicina ${ }^{24}$,

Os aminoglicosídeos concentram-se diversas vezes no túbulo proximal, diferentemente do que ocorre no distal. Quando atinge as células do túbulo proximal o aminoglicosídeo que foi livremente filtrado, por pinocitose (pelo lado luminal ) e por captação (pelo lado basolateral), utilizam mecanismos de secreção transtubular de aminoglicosídeo quase desprezivel do ponto de vista da nefrotoxicidade 25

A gentamicina (como outros aminoglicosídeos ) liga-se dentro da célula aos lisossomos e formam corpos mielóides, aumentando sua presença, induzindo fosfolipidose e reduzindo intensamente a função das fosfolipases locais; isto facilita a liberação de aminoglicosídeo para o citoplasma, onde haverá interferência na cascata de fosfatidil-inositol, bloqueando a hidrólise da fosfolipase $\mathrm{C}$ e afetando assim toda a cascata de sinalização intracelular e sua regulação por estímulos e bloqueios endógenos e exógenos ${ }^{25}$, alterando intensamente a homeostase celular,

Além das interações com os lisossomas há provável atuação da gentamicina no estresse oxidativo sobre as mitocôndrias, presumivelmente devido sua capacidade de catalizar a formação de radicais livres'.

Desta forma, a meia vida local da droga é bastante prolongada 18 (por meses após a interrupção da terapia), devido o acúmulo intracelular 25 . Outros estudos demonstram ainda que um maior acúmulo tecidual da droga pode ocorrer em conseqüência de administrações múltiplas, comparando às terapias de infusão única ${ }^{14,25}$

Resultados de pesquisas referentes a sua fisiopatologia afirmam que a IRA nefrotóxica induzida por aminoglicosídeos pode ser intensificada quando associada à fatores como: contrastes, antiinflama-tórios não hormonais, anestésicos e efeito direto de toxinas bacterianas $12,14,24$

O conhecimento de variáveis como a dose utilizada, duração do tratamento, determinação do nível de pico da dose e do seu nível sérico basal além do intervalo de administração, pode possibilitar um tratamento com redução dos riscos, embora alguns trabalhos demonstrem ausência de correlação entre a toxicidade renal e tais fatores ${ }^{4,15,17}$

Dentre elas, a adoção de doses únicas diárias é uma alternativa que tem sido investigada visando a redução do risco nefrotóxico da gentamicina 9,10,11,16,21 bem como a sua praticidade $7,15,19$ e vantagem econômica 4,13,15,19 sem alterar a eficácia 4,18,19 Outros estudos sugerem entretanto a monitorização $3,12,16$ ou ajuste da dose ${ }^{6}$, como a melhor estratégia de controle da toxicidade do fármaco.

Os índices de nefrotoxicidade ainda vinculados à droga são alarmantes e reiteram maiores investimentos na busca por esclarecimentos a respeito de estratégias de manuseio que possam representar medidas de atenuação da toxicidade ${ }^{2}$

\section{OBJETIVO}

- Padronizar o modelo de insuficiência renal aguda pela gentamicina no laboratório experimental da Escola de Enfermagem do USP.

- Avaliar a interferência do intervalo de infusão na nefrotoxicidade da gentamicina, 


\section{MATERIAL E MÉTODOS}

Foram utilizados ratos machos, da raça Wistar, adultos, pesando entre 250-300g, agrupados da seguinte forma:

- Grupo 1: grupo controle. Animais que receberam solução salina, que é veículo da gentamicina (genta) $(0,1$ $\mathrm{ml} /$ intra peritoneal (i.p.)/ 5 dias).

- Grupo 2: grupo genta $1 \mathrm{x}$ dia. Animais que receberam genta (100 mg/ kg peso/ 1 x dia/ i.p./ 5 dias).

- Grupo 3: grupo genta $2 \mathrm{x}$ dia. Animais que receberam genta (50 mg/ kg peso/ 2 x dia/ i.p./ 5 dias). alimento.

Todos os animais tiveram livre acesso à água e

Cada animal ao final do protocolo, oú seja, no $5^{\circ}$ dia, foi colocado em gaiola metabólica, com vistas à coleta da urina daquele período (24hs), para a verificação do clearance de creatinina, que serviu como indicador de função renal. Após serem retirados das gaiolas metabólicas, foram submetidos à laparotomia para realização de coleta de sangue, com punção da artéria aorta.

A fórmula do clearance de creatinina é $\mathrm{Cl}_{\mathrm{cr}} \underset{-\mathrm{f} \text { it }}{\mathrm{C}} \mathrm{V}$ onde: $\mathrm{Cr}=\underset{\mathrm{u}}{\mathrm{c}} \mathrm{reatinina}$ urinária

$\mathrm{Cr} r$

$$
\begin{aligned}
& \mathrm{V}=\text { volume urina /minuto } \\
& \mathrm{Cr}=\text { creatinina sérica }
\end{aligned}
$$

Assim, foram efetuadas as dosagens de creatinina séricas e urinárias, que serviram como indicadores da função renal. O método utilizado para as dosagens foi fluorimetria23.

Foi utilizado o teste de Kruskal-Wallis, comparações múltiplas de Dunn, para as comparações estatísticas entre os diversos grupos.

Todos os procedimentos, tratamentos e dosagens, foram realizados no Laboratório Experimental da Escola de Enfermagem da Universidade de São Paulo.

\section{RESULTADOS E DISCUSSÃO II}
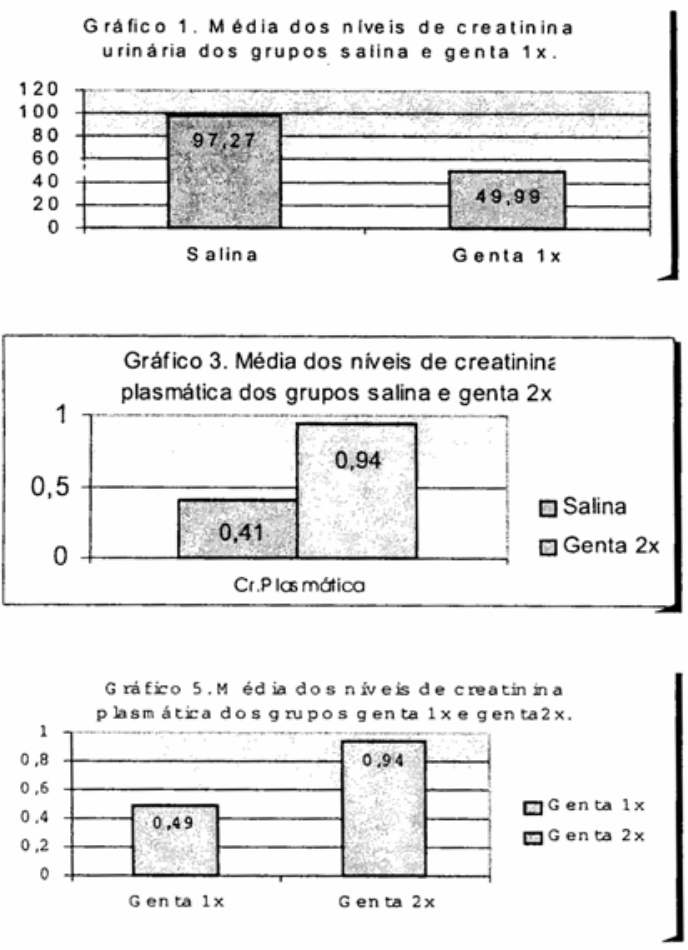
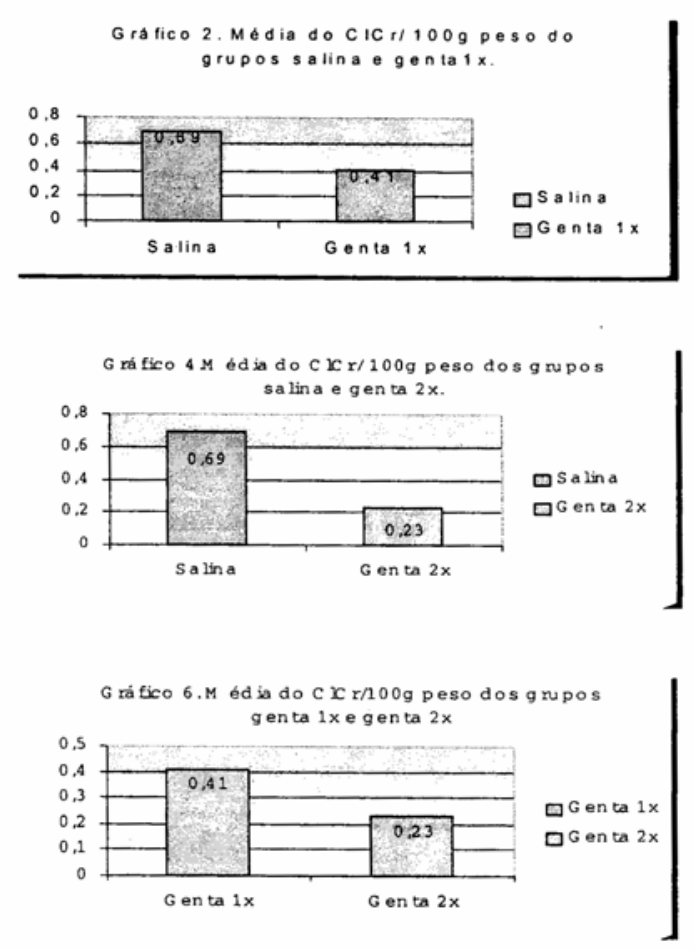

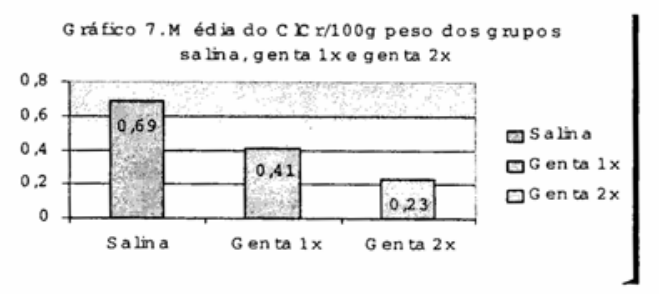


Os dados obtidos na análise estátistica, constatam que as diferenças de peso, volume urinário de vinte e quatro horas e volume urinário por minuto dos animais nos diferentes grupos, não foram significantes entre si, portanto não serão apresentados.

Os demais resultados deste estudo demonstram que, comparando o grupo de animais que receberam dose única (lx) de gentamicina (genta )/100mg/dia com o grupo salina (controle), observa-se uma diminuição significante da creatinina urinária do primeiro grupo quando comparada ao segundo (considerado dentro dos padrões de normalidade da função renal) $(p<0,05$ Gráfico 1) e, embora não haja diferença significante na dosagem de creatinina plasmática, o clearance de creatinina/ $100 \mathrm{~g}$ peso, adotado como marcador de função renal neste estudo, indica um declínio substancial da filtração glomerular dos animais tratados com dose única ( $<<0,05$ - Gráfico 2). Este fato confirma a nefrotoxicidade da gentamicina 25 .

Os animais que receberam a dose fracionada (2x) de genta $100 \mathrm{mg} /$ dia, ao serem comparados com o grupo controle demonstram um declínio na dosagem de creatinina urinária semelhante aos animais que receberam dose única, o que indica como já foi dito, redução da filtração glomerular. Entretanto, somandose a este fato, verifica-se um importante aumento na creatinina plasmática do grupo genta $2 \mathrm{x}$, indicando perda acentuada de função renal com consequente acúmulo de compostos nitrogenados no organismo $(\mathrm{p}<0,05$ - Gráfico 3).

Comparando o clearance de creatinina $(\mathrm{C} 1 \mathrm{Cr}) /$ $100 \mathrm{~g}$ peso, observamos que os animais tratados com genta fracionada tiveram um resultado de $\mathrm{C} 1 \mathrm{Cr}=0,23$ contra $\mathrm{C} 1 \mathrm{Cr}=0,69$ do grupo controle $(\mathrm{p}<0,05$ - Gráfico 4), este dado sugere que devido à meia vida local da droga ser bastante prolongada, seu fracionamento contribui para um maior acúmulo tecidual, provocando profunda alteração da homeostase celular devido sua interferência em vários mecanismos, como indução de fosfolipidose, interferência na cascata de fosfatidil-inositol e consequentes alterações na sinalização intracelular, bem como a catalização de radicais livres, entre outros, caracterizando consequente insulto renal 1,25 , conforme comentado na introdução deste relatório.

Não foi observada diferença significante na dosagem da creatinina urinária dos grupos genta $1 \mathrm{x}$ e genta $2 \mathrm{x}$. Por outro lado, o grupo genta $2 \mathrm{x}$ revelou acentuada elevação na creatinina plasmática $(p<0,05$ Gráfico 5) .

A alteração na creatinina plasmática do grupo genta $2 \mathrm{x}$, comparado ao grupo genta $\mathrm{x}$, parece ser $\mathrm{o}$ principal determinante da redução do clearance de creatinina observado no primeiro grupo $4,14,25$ ( $p<0,05$ - Gráfico 6). Este achado reitera dados anteriormente publicados.

\section{CONCLUSÕES}

A implementação permitiu verificar que o modelo de IRA nefrotóxica pela gentamicina foi padronizado no nosso laboratório uma vez que os dados obtidos foram semelhantes àqueles acessados em literatura.

$O$ estudo concluiu também que o esquema de infusão única de gentamicina determinou menor nefrotoxicidade quando comparado ao grupo que recebeu a mesma dose de forma fracionada.

$\mathrm{O}$ regime de dose única mostra vantagens quanto ao efeito nefrotóxico, sugerindo também outros benefícios como redução do custo ( diminuição no tempo de prestação de serviços de enfermagem,

materiais utilizados para administração da droga e gastos decorrentes do acometimento por IRA), e segurança quanto à eficácia terapêutica, colaborando na redução dos altos índices de mortalidade relacionados com esta lesão.

\section{REFERÊNCIAS BIBLIOGRÁFICAS:}

1.BALIGA, R.; UEDA, N.; WALKER, PD.; SHAH S.V. Oxidant mechanisms in toxic acute renal failure. Am J Kidney Dis., v. 29 , n.3, p. $465-77,1997$.

2.BARCLAY, M.L.; BEGG, E.J.; HICKLING, K.G. What is the evidence for once-daily aminoglycoside therapy? Clin Pharmacokinet., v. 27, n.1, p. 32-48, 1994.

3.BARCLAY, M.L.; KIRKPATRICK, C. M.; BEGG E.J. Once daily aminoglycoside therapy. Is it less toxic than multiple daily doses and how should it be monitored? Clin Pharmacokinet., v.36, n.2, p. 89-98, 1999.

4.BLASER, J. Dosing aminoglycosides once a day. Schweiz Med Wochenschr, v. 76, p. 39S-48S, 1996

5.BURDMANN, E.A. e cols. Epidemiologia, In: SCHOR, N.; BOIM, M.A.; SANTOS, O.F.P. Insuficiência Renal Aguda: Fisiopatologia, Clinica e Tratamento. Sarvier, la ed., cap.1, p. 1-7 São Paulo, 1997.

6.CHRISTENSEN, S.; LADEFOGED, K.; FRIMODT-MOLLER, N. Experience with once daily dosing of gentamicin: considerations regarding dosing and monitoring. Chemotherapy. , v.43, n.6, p. 442-50, 1997.

7.CHRISTENSEN, S.B.; JENSEN, K.M.; RYSGAARD, K.; LADEFOGED, K; FRIMODT MOLLER, N. Experience with daily single dose administration of gentamicin. Ugeskr Laeger. , v.159, n.21, p. 3167-71, 1997. 
8.DEAMER, R.L.; DIAL, L.K. The evolution of aminoglycoside therapy: a single daily dose. Am Fam Physician, v. 53, n.5, p. 1782-6, 1996.

9.DILLON, K.R.; DOUCHERTY, S.H.; CASNER, P.; POLLY, $S$. Individualized pharmacokinetic versus standard dosing of amikacin: a comparison of therapeutic outcomes. J Antimicrob Chemother., v. 24, n.4, p.581-9, 1989.

10.FERRIOLS-LISART, R.; ALOS-ALMINANA, M. Effectiveness and safety of once-daily aminoglycosides: a meta-analysis. Am $\boldsymbol{J}$ Health Sys Pharm, v.53, n.10, p. 1141-50, 1996.

11.GILBERT, D.N. Once-daily aminoglycoside therapy,. Antimicrob Agents Chemother., v. 35. n.3, p.399-405, 1991.

12.GRAUER, G.F. Prevention of acute renal failure, Vet Clin North Am Small Anim Pract., v. 26, n.6, p.1447-59, 1996.

13.HITT, C.M.; KLEPSER, M.E.; NIGHTINGALE, C.H.; QUINTILIANE, R.; NICOLAU, D.P. Pharmacoeconomic impact of once-daily aminoglycoside administration, Pharmacotherapy., v.17, n.4, p. 810-4, 1997.

14.LEEHEY, D.J.; BRAUN, B.I.; THOLL, D.A.. ; CHUNG, L.S.; GROSS, C.A.; ROBACK, J.A..; LENTINO, J.R. Can pharmacokinetic dosing decrease nephrotoxicity associated with aminoglycoside therapy. J Am Soc Nephrol., v.4, n.1, p.81-90, 1993.

15.LUND, E.S.; ELIASEN, P.; WANDALL, E.P.; HANSEN, E.E; LOMHOLDT, J.D.; FRIIS, H.M. Aminoglycoside treatment I. Administration of gentamicin once versus three times daily. Ugeskr Laeger., v. 160, n.1, p.45-9, 1997.

16.MCLEAN, A..J.; IOANNIDES-DEMOS, L.L.; SPICER, W.J.; CHRISTOPHIDIS, N. Aminoglycoside dosing: one, two or three times a day?_Med J Aust., v.164, n.1, p. 39-42,
1996.

17.MOORE, R.D.; SMITH, C.R.; LIPSKY, J.J.; MELLITS, E.D.;

18.MUNCKHOF, W.J.; GRAYSON, M.L.; TURNIDGE, J.D. A meta-analysis of studies on the safety efficacy of aminoglycosides given either once daily or as divided doses. J Antimicrob Chemother., v. 37, n.4, p. 645-63, 1996.

19.NICOLAU , D.P.; FREEMAN, C.D.; BELLIVEAU, P.P.; NIGHTINGALE, $\quad$ C.H.; ROSS, J.W.; QUINTILIANI, R. Experience with a once-daily aminoglycoside program administered to 2,184 adult patients. Antimicrob Agents Chemother., v. 39, n.3, p. 650-5, 1995

20.NISSENSON, A..R. Acute renal failure: definition and pathogenesis, Kidney Int Suppl. v. 66, p.S7-10, 1998.

21.PERITI, P. Preclinical and clinical evaluation of oncedaily aminoglycoside chemotherapy J Chemother., v. 7, n.4,.p. 31137, 1995.

22.RODMAN, D.P.; MAXWELL, A..J.; McKNIGHT, J.T. Extended dosage intervals for aminoglycosides. Am $\boldsymbol{J}$ Hosp Pharm., v. 51, n.16, p.2016-21, 1994.

23.SMITH, H.W.; FILKELSTEIN, N.; ALIMINOSA, L.; CRAWFORD, B.; GRABER, M. The renal clearancs of substituted hippuric acid derivatives and other aromatic acids in dog and man, J.Clin. Med., v. 24, n. 388-404, 1945.

24.SANTOS, O.F.P. Participação do fator ativador de plaquetas em alguns modelos de nefrotoxicidade em ratos. Tese apresentada à Escola Paulista de Medicina para obtenção do título de doutor em medicina nefrologia. São Paulo, 1990.

25.VATTIMO, M.F.; SANTOS, O.FP.; SCHOR, N.; BOIM, M.A.. IRA nefrotóxica: antibióticos e antivirais. In: SHOR, N.; BOIM, M.A..; SANTOS, O.F.P. Insuficiência renal aguda - fisiopatologia, clinica e tratamento. Sarvier, 1' edição, cap.14, p.103-108. São Paulo, 1997. 